\title{
RESOLUCIÓN DE PROBLEMAS Y CREATIVIDAD: IMPLICACIONES PARA EL CURRÍCULO DE CIENCIAS (*)
}

\author{
GARRET, R.M.
}

School of Education, University of Bristol, U.K.

II Congreso Internacional Sobre Investigación en la Didáctica de las Ciencias y de las Matemáticas. Valencia, 1987.

$\left({ }^{*}\right)$ Este trabajo forma parte de un proyecto de investigación conjunta entre la Universidad de Valencia y la Universidad de Bristol, dentro del Programa de Acciones Integradas Hispano-Británicas.

Versión de Joaquín Martínez Torregrosa.

\section{SUMMARY}

This paper deals, mainly, with three questions: why is problem solving seen as an essential activity to be enhanced in schools? What can we understand by problem solving? and, which are the implications for science teaching of a problem solving orientation?

En este articulo trataré, muy brevemente, tres cuestiones principales: ¿por qué la resolución de problemas se considera cada vez más como una actividad importante que debe ser fomentada en las escuelas? ¿Qué debemos entender por resolución de problemas? Por último, ¿qué implicaciones se derivan para la enseñanza de las ciencias cuando se adopta un enfoque basado en la resolución de problemas? (ver, por ejemplo, Closset et al 1982; Gil y Martínez-Torregrosa, 1983).

Ciertamente en el Reino Unido y Europa el lugar de la resolución de problemas dentro del conjunto del currículo está siendo mucho más resaltado que hasta ahora, y los cambios actuales que se están haciendo en el currículo de ciencias, inglés y en el galés colocan dicha actividad en un lugar central.

\section{1. ¿POR QUÉ ES IMPORTANTE LA RESOLU- CIÓN DE PROBLEMAS?}

Hay una antigua y ampliamente sostenida creencia de que resolver problemas es una actividad fundamental de la ciencia (por ejemplo, Blough (1942), Stollberg (1956), y Turner (1957)). Este primer movimiento estuvo en gran medida vinculado al lamado aprendizaje por «descubrimiento» y fue por consiguiente visto como una manera adecuada de conseguir que los niños hicieran las cosas por sí mismos y aprendieran de un modo que se esperaba que fuera más significativo. Encontrando sus propias soluciones a los problemas, se argumentaba, los niños podrian aprender las cosas haciéndolas y esto haría entonces más probable que las recordaran. Por algún tiempo este enfoque cayó en descrédito. Se consideró como un juego desestructurado y una pérdida de tiempo, y la idea de que los niños podian «redescubrir» por sí mismos todo lo que necesitaban saber, como evidentemente no realista. Más recientemente se ha hecho hincapié en la enserfanza de las ciencias como un proceso más que como una serie de informaciones, hechos, modelos y teorías que deben ser aprendidas. Este movimiento general, no sólo en las ciencias, hacia un currículo orientado a los procesos está asimismo basado en un número de consideraciones:

1. El enorme crecimiento del conocimiento supone tal presión en un currículo tradicional orientado a la información que lo hace no solamente abarrotado sino impracticable. Un currículo basado predominantemente en la información es, simplemente, insostenible.

2. No solamente la información crece con rapidez creciente, sino que cambia, y lo que es relevante a la sociedad hoy es de interés periférico mariana.

3. Aspectos ético/filosóficos también deben salir a la luz. Si presentamos la ciencia honesta y fielmente, no como un conjunto de verdades fijas, sino como un conjunto constantemente cambiante de las mejores explicaciones existentes en el momento, entonces debemos mostrar a nuestros estudiantes cómo 
cambia la ciencia y qué es lo que hacen los cientifi$\cos$; qué hace distinguible la actividad científica de otro tipo de actividades. A este respecto, los trabajos de Popper y Kuhn han sido muy influyentes ( $p$. ej. Brandon, 1981).

4. Argumentos similares son formulados por los integracionistas (Brown, 1977) pero posiblemente el más importante de todos sea el formulado por Bernstein (I971) que defiende que los procesos fundamentales básicos o estructuras profundas de cualquier materia (ciencias incluidas) deben salir a la luz desde el mismo principio de la enseñanza; una apertura del jardín secreto en vez del levantamiento de barreras para el aprendizaje.

5. Consideraciones de lo que debería constituir el núcleo (core) de los currículos han conducido también a los expertos de la educación a cambiar desde el masivo e inmanejable conjunto de información que rodea a las ciencias hacia la consideración de un más manejable y potencialmente más útil conjunto de procesos.

Todos estos argumentos a favor de un enfoque de procesos incluye como un elemento principal el proceso de resolución de problemas. Como es bastante obvio, se trata también de una actividad muy importante en la vida cotidiana; una de las así llamadas destrezas para la vida que ahora se consideran como características fundamentales de los modernos currículos escolares. La actividad diaria y el trabajo profesional supone enfrentarse a problemas. También es una actividad representativa en los campos específicos de la ciencia y ta tecnologia; incluso algunas veces es considerada (erróneamente) como la actividad definitoria esencial de la ciencia; la característica que distingue la ciencia de otras ocupaciones. Tiene una importancia personal, profesional y económica y consecuentemente se trata de una actividad que no puede ser minusvalorada en los currículos escolares.

No sólo los teóricos del currículo predican que la resolución de problemas es una destreza importante que debe ser fomentada (p. ej. Barnes, 1982), los investigadores del currículo (Eggleston, Galton \& Jones, 1975) encuentran que es una característica fundamental del modo en que los profesores abordan su tarea y que los propios profesores la consideran como algo que debe ser incorporado en las actividades de aprendizaje de sus alumnos (p. ej. Wilson, 1977, y Aylesworth, 1965).

Quizás, en el fondo, la razón principal para este importante cambio en énfasis y en direccion hacia la resolución de problemas en particular, como uno de los procesos principales que han de desarrollarse, es un descontento general con actividades curriculares tradicionales y con la importancia dada hasta ahora a los profesores y la enseñanza. El cambio radical que está ocurriendo es un traslado hacia el que aprende y el aprendizaje. Del mismo modo en la investigación podemos detectar también una insatisfacción respecto a las simples descripciones de la transacción enseñanza/aprendizaje frente a cualesquiera intentos coherentes de explicar los procesos reales de aprendizaje que están teniendo lugar.

Si esto es así y necesitamos examinar al que aprende y su actividad, entonces chocamos con el concepto de «pensar» casi inmediatamente: esa actividad privada, a menudo inefable, que tiene lugar dentro de la cabe$z a$, difícil de estudiar e igualmente difícil de manejar en la clase.

\section{QUÉ ES RESOLVER PROBLEMAS}

A menudo, pensar es considerado casi como sinónimo de solucionar problemas; Humphrey (1951), por ejemplo, dice que pensar es:

«lo que ocurre cuando un organismo... encuentra, reconoce y soluciona un problemas.

No obstante, si insistimos en que solucionar problemas es un elemento necesario dentro de la definición de pensar, entonces estamos considerando que cualquier actividad que falle en encontrar la solución cae fuera del campo del pensamiento. Pero jes suficiente la mera ausencia de una solución -inevitablemente el útimo acto de un proceso con frecuencia largo y prolongadopara calificar todo lo que se ha hecho antes como nopensamiento?

Este énfasis en la solución, en obtener una solución, es desafortunado y posiblemente un término mejor que podria usarse y que he defendido en otras ocasiones (Garrett, 1987), es enfrentarse a problemas. Esta denominación posiblemente describe más fielmente la actividad en una situación problemática sin implicar necesariamente el acto final de la solución. Incluso, como veremos más tarde, las soluciones no son siempre posibles, y una preocupación o énfasis en las soluciones es algo que debemos explorar más adelante. No obstante, antes de que lo hagamos, examinemos con más detalle la idea global de hallar soluciones.

Tener éxito, es decir hallar una solución a un problema, es un acto productivo. Ha resultado algo positivo. En efecto, solucionar problemas ha sido descrito como pensamiento creativo. A menudo consideramos al cientifico creativo como aquel que hace surgir la respuesta el que produce soluciones a problemas. Habjtualmente la palabra «creativo» significa producir ofabricar. Con esta acepción, desde luego, la solución de un problema solamente puede ser descrita como creativa, o como dice Debney (1971), solucionar problemas es pensar creativamente. Pero la creatividad es más que simplemente producir una respuesta. Existen otros criterios para juzgar la creatividad.

Examinando este concepto un poco más cuidadosamente vemos que otros factores son considerados impor- 
tantes. Bruner (1962), por ejemplo, considera lo imprevisto (surprise) como un importante factor de cualquier acto creativo. Parves y Brunelle (1967) requieren la novedad, al igual que el valor o la utilidad. Stein (1953) también considera la novedad importante, mientras que Rogers (1959) usa las palabras «incomparables, único» en relación a la creatividad.

Como sugiere Mednick (1962), «el proceso de pensamiento creativo (se define como) la formación a partirà de elementos asociativos de nuevas combinaciones que o bien responden a requerimientos especificos o son de alguna manera útiles».

Así, podría considerarse que la creatividad supone novedad u originalidad y utilidad (Garrett, 1984). Como he sugerido en otra parte (Garrett, 1987), esto puede ser expresado como se indica en la figura 1.

figura /

La relación entre originalidad, utilidad y creatividad.

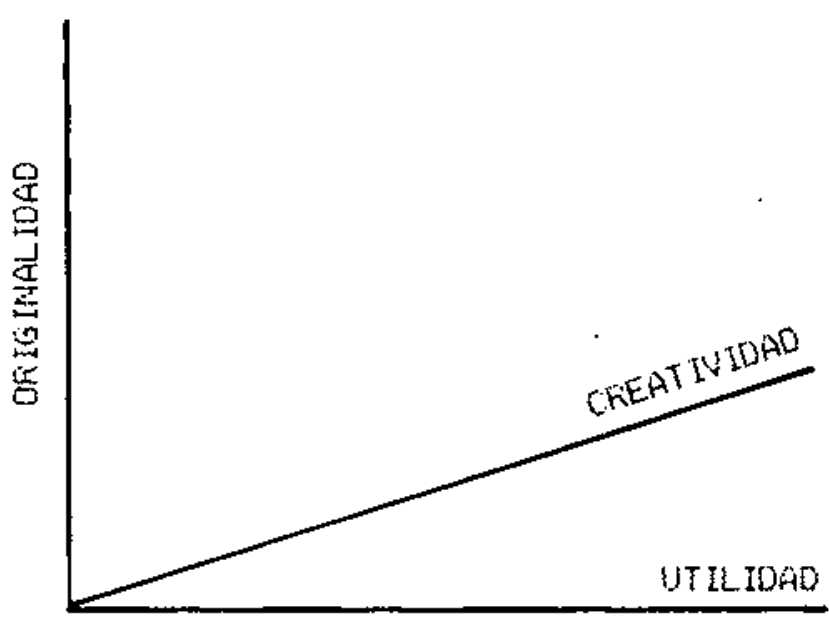

Dicha relación sugiere por consiguiente que una resolución de problemas de máxima creatividad debe incluir utilidad y originalidad en cualquier solución. Si se pone el énfasis en la utilidad (siendo mínima la orjginalidad) entonces la creatividad se reduce. Igualmente, ideas altamente originales pero en gran medida inutiles no son particularmente creativas. En la situación de enfrentar verdaderos problemas se requiere un máximo de creatividad y esto tiene implicaciones para el modo en que preparamos a nuestros estudiantes y pa. ra el currículo que les suministramos, un punto que recogeremos más adelante.

Resumiendo brevemente, la posición que puede man. tenerse es ésta:

1. Solucionar problemas es parte del proceso de pensar y este incluye todas las acciones del «enfrentamiento de problemas» e incluso el reconocimiento de que existe un problema.
2. La actividad de enfrentar problemas puede ser más o menos creativa dependiendo del grado de utilidad y originalidad que incluya.

Estos puntos son de importancia en cualquier discusión del desarrollo de los nuevos currículos de ciencias pero antes de hacer esto, es necesario clarificar aún más lo que realmente supone el concepto de resolver o enfrentar problemas. Es necesario discutir cuatro cuestiones básicas:

1. ¿Existen tipos distintos de problemas?

2. ¿Todos los procesos de resolución son iguales?

3. ¿Hay diferentes tipos de resolventes?

4. ¿Los problemas son fijos e independientes de su contexto y del resolvente?

Desde hace tiempo ha existido una diferenciación en tre los llamados problemas «abiertos» y «cerrados» (p. ej. Holt, 1969).

Problemas cerrados son aquellas situaciones que tienen bien sólo una respuesta o más de una, pero igualmente correctas. El resolvente generalmente sabe cuándo ha llegado a una respuesta y, como sabemos que hay una respuesta a la que llegar, entonces es posible solucionar estas situaciones, p. ej. icuánta alfombra necesito para cubrir el suelo de esta habitación?

Hay, por otro lado, situaciones para las que puede haber varias respuestas de las que ninguna de ellas sea correcta o equivocada en términos absolutos, sino simplemente la más adecuada para un conjunto dado de circunstancias. Posiblemente están involucrados un conjunto de factores en conflicto y nunca podemos estar seguros de que hayamos llegado siquiera a la mejor respuesta. Estas situaciones abiertas carecen de una solución definida y solamente pueden ser resueltas, $\mathrm{p}$. ej.: ¿Qué calidad de alfombra debo poner en esta habitación?

Las dos situaciones anteriores tienen cierta similitud. Se pueden solucionar potencialmente dentro de un paradigma dado o resolverse dando información suficiente. Igualmente, ambas resaltan la utilidad. De hecho la componente originalidad de la creatividad puede en ocasiones ser un estorbo para su solución (Garrett, 1984).

A todas las situaciones de este tipo, las he llamado rompecabezas (puzzles) (Garrett, 1984, 1987). Esta denominación no pretende degradarlas en alguna medida o sugerir que los rompecabezas son más simples que los problemas. De hecho, Kuhn (1964) ha sugerido que toda la actividad cientifica normal es una actividad de solucionar rompecabezas desarrollada dentro de los paradigmas vigentes.

Hay otro grupo de situaciones enigmáticas, no obstante, que no son ni solucionables ni resolubres; son solamente comprensibles. A este tipo los he llamado «problemas verdaderos»». Ellos requieren que el resolvente salga de los paradigmas existentes, los reaplique, los 
reinterprete o, en último término, que produzca un paradigma totalmente nuevo. El reconocimiento y com. prensión de una situación de este tipo da lugar a nuevos rompecabezas que deben ser resueltos o solucionados.

De modo bastante claro, si aceptamos estas ideas no todo "proceso de solución es igual, se requieren diferentes destrezas, enfoques y capacidades según estemos enfrentándonos a rompecabezas abiertos o cerrados o a verdaderos problemas.

Podemos ir más allá y aventurar que las situaciones que despiertan el mayor interés son las que caen en nuestra frontera personal entre rompecabezas y problema (ver figura 2). Hay por consiguiente una zona de inte- rés óptimo y es más probable que el trabajo en esta zona produzca aprendizaje significativo, en el sentido de que el material aprendido encajará en la red cognoscitiva ya existente en el que aprende. Más que generar información, por tanto, el que aprende habrá adquirido conocimiento. Si a las personas que aprenden se les pide que trabajen fuera de su zona personal de interés óptimo, serán bien incapaces de realizar la tarea, sintiéndose en el área desconocida, sin tener con qué trabajar y siendo, incluso, incapaces de reconocer como problemas las situaciones que se les presentan, o bien, en el extremo opuesto, los rompecabezas empezarán a ser cada vez menos interesantes según nos alejemos de la zona de interés óptimo, y, como mucho, suministrarán información de poco valor.

figura 2

Relación mutua entre probłemas y rompecabezas y de éstos respecto al conocimiento.

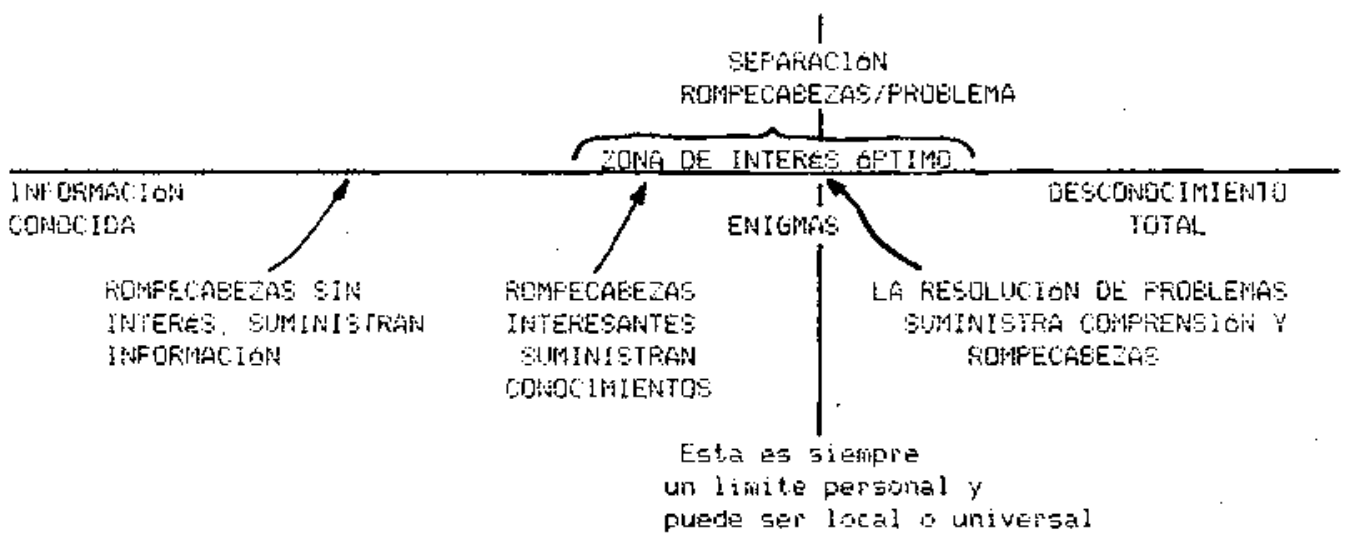

La separación rompecabezas/problema variará dentro de un individuo y entre individuos distintos, dependiendo de sus intereses y conocimientos previos. Así, algunos actuarán en el limite universal, o en las llamadas fronteras del conocimiento, en un área particular; la mayor parte, sin embargo, tendrá fronteras personales muy alejadas del limite universal. Igualmente importante de advertir es una diferencia individual adicional. Distintos tipos de resolventes preferirán distintas áreas de actividad, o incluso trabajar en problemas o en rompecabezas. Dichas diferencias individuales pueden ser explicadas por los distintos estilos cognoscitivos (Ronning et al, 1984; Garrett, 1984), Del mis* mo modo, la percepción por los individuos del contexto en el que se integra la situación puede diferir y la situación enigmática, rompecabezas o problemas, puede tomar diferentes características.

\section{ALGUNAS IMPLICACIONES PARA EL CU- RRÍCULO DE CIENCIAS}

¿Cuáles son entonces las principales implicaciones de estas ideas para el curriculo de ciencias? Para empezar, revisemos las primeras dos cuestiones que hemos planteado sobre los problemas; ihay distintos tipos de problemas? y itoda resolución de problemas es de la misma naturaleza? Si se acepta la distinción hecha en este artículo -que se puede distinguir entre rompecabezas y problemas - entonces es posible ver que requieren diferente actividad intelectual. La resolución de rompecabezas requiere la producción de respuestas correctas o más apropiadas; en consecuencia, se están buscando metas útiles. El mayor hincapié se hace en la componente «utilidad» de la creatividad y como consecuencia los distintos "trucos», «métodos", algoritmos, etc, para solucionar son las destrezas que deben resaltarse. Con mucha frecuencia, nuestras clases de ciencias, cuando nos salimos del suministro de información, están llenas de actividades de solución de rompecabezas (generaimente de la variedad cerrada). Incluso entonces, mucho de lo que se hace consta de rompecabezas superficiales bien alejados de la zona de interés óptimo, lo que, como he argumentado aqui, so* lamente sirve para generar información que, en el mejor de los casos, es de poco interés, y en el peor, no tiene sentido. El tipo de resolución de rompecabezas que se hace en las escuelas, por tanto, debe ser un mayor rango. Debería estar dentro de las zonas de interés óptimo de los alumnos, - preferiblemente rompecabezas propuestos por ellos mismos-, de este modo sería posible que actuaran, al menos durante parte de su tiempo, como científicos normales en el sentido kuh. niano del término. 
No obstante, cambiar hacia una resolución más exigente de rompecabezas no es suficiente en si mismo. No sólo no contesta a todas las criticas hechas al cur rículo basado en la solución de rompecabezas; particularmente hace poco, si es que hace algo, por cambiar los sutiles pero muy fuertes mensajes adicionales que cualquier curriculo basado en la solución de rompecabezas puede estar transmitiendo. Este currículo oculto puede ser resumido muy brevemente así; "Espera siempre respuestas a los problemas" (y por implicación, la ciencia siempre produce respuestas) y ulas únicas cuestiones que vale la pena plantearse son aquellas que puede ser constestadas como correctas o erróneas"y.

Además de no tratar adecuadamente los aspectos de aburrimiento y el curriculo oculto, si seguimos una dieta estricta de resolución de rompecabezas cerrados, corremos el riesgo de olvidar ta originalidad de los alumnos, -incluso hasta el extremo de suprimirla (Garrett, 1984)-, y, del mismo modo, es mínima la comprensión sobre la naturaleza de la ciencia. Se debe encontrar un equilibrio, suministrando más rompecabezas abiertos y verdaderos problemas en los que alumnos y profesores no conozcan necesariamente los resultados y donde el «éxito» no sea absoluto. El suministro de dichas experiencias supondrá al menos los siguientes progresos: (1) se fomentarán verdaderos intentos de llegar a una comprensión real de los aspectos planteados; (2) se podrá ejercer la originalidad; (3) se fomentará una verdadera formulación de hipótesis; (4) se generarán rompecabezas que automáticamente caerán en la zona de interés óptimo; (5) se fortalecerá una actitud mucho más abierta, flexible y realista hacia los logros de la ciencia y se apreciarán las limitaciones del proceso cientifico; y (6) se logrará una enseñanza a través de, en vez de al margen de, los procesos científjcos. Este último provecho será en los dos sentidos, tanto en el sentido en que Kuhn ve la ciencia, con los alumnos intentando articular los paradigmas vigentes, como en el sentido popperiano, en el cual se incitaría a los estudiantes a plantear hipótesis contrastables.

Dado que la variedad en las situaciones de enseñan$\mathrm{za}$ /aprendizaje es importante para acomodarse a las diferencias entre profesores y alumnos, lo que se propone aquí es valioso porque colocará a ambos participantes en el proceso de aprendizaje en diferentes situaciones, permitiendo asi que las diferencias se desarrollen y exploten en bien de todos los implicados. De nuevo, es importante que el contexto en el que se plantea el problema sea variado para acomodarse a las posibles diferencias en el estilo de aprendizaje. Cierta evidencia (Ronning et al, 1984 y Garrett, 1984) sugiere que el modo de presentación puede ser importante para el camino que sigue el resolvente. Situaciones de lápiz y papel, planteamientos prácticos, situaciones estructuradas, guiadas o sin estructurar, son, todos ellos, contextos posibles para plantear el "enfrentamiento» de problemas, y que pueden ser importantes para influenciar diferentes actividades y resultados en diferentes

\section{estudiantes.}

Hay una influencia del contexto, posiblemente más sutil y probablemente no tan bien admitida, que puede tener pronfundos efectos sobre la actitud hacia la ciencia que se crea en un individuo, y que es el planteamiento global del currículo de ciencias. Ya se ha aludido a ello anteriormente en este artículo. La idea de que los profesores transmiten mensajes ocultos sobre que los problemas siempre se pueden solucionar, que hay caminos correctos y erróneos de resolver problemas (el llamado método científico) y, por inferencia, la idea de que la ciencia siempre puede solucionar rompecabezas y problemas. Además también se está transmitiendo la idea de que la ciencia es un método invariable para solucionar rompecabezas y problemas, que también permanecen, en gran medida, invariable. Esto, desde luego, no es así. Los rompecabezas, y en particular los problemas a los que se enfrentaron Galileo y Newton no son los mismos a los que hoy se enfrentan los científicos. Desde luego, no en el sentido de la especificidad de los fenómenos que estuvieron investigando, que obviamente no son objetivos de la física actual, excepto en formas más refinadas, sino en el sentido de que las mismas cuestiones que podian y no podían ser preguntadas sobre los fenómenos y el modo en que podian abordarse eran diferentes. Sus empresas científicas no podian separarse del contexto socia!, económico, político y religioso en que estaban inmersos. De hecho, este mismo medio social en el que se planteaban sus problemas dictaban en gran medida el modo en que podian pensar sobre ellos -incluso cuáles eran los problemas importantes a tratar.

Podría parecer que hay dos maneras básicas en que la ciencia es enseñada en las escuelas. Primero está la transmisión tradicional de información, la cual, cuando se examina cuidadosamente, es poco más que una historia enlatada del desarrollo de los conceptos científicos. En segundo lugar, está el enfoque actual favorecedor de los procesos con el que esperamos ayudar a que los estudiantes piensen y actúen como científicos. Nos equivocamos.

El examen hecho aqui de uno de los principales procesos del pensamiento científico, el de enfrentarse a pro. blemas y, en particular, una consideración de su planteamiento contextual, lleva a admitir tres posibles perspectivas desde las que presentar la resolución de problemas, cada una de las cuales cambiará necesariamente la misma naturaleza de los rompecabezas/problemas. Estas se pueden exponer simplemente como: (1) los procesos de resolución de problemas actuales; qué es lo que preocupa hoy a los científicos y cómo plantean sus tareas; las cuestiones que preguntan y las técnicas que emplean: (2) los procesos pasados, qué hicieron los científicos en otros tiempos; cuáles fueron sus intereses y problemas; cómo trataron de contestarlos y por qué; (3) los procesos históricos: cómo han cambiado las ideas, preguntas y técnicas a lo largo de los años, 
y cómo -en ocasiones específicas- se han juntado las ideas para ayudar a resolver o comprender aspectos desconocidos o para producir situaciones sin respuesta donde nunca antes habian existido.

Las tres perspectivas tienen que jugar un importante papel en nuestra enserianza de las ciencias y es urgente que prestemos atención a estos respectos. Uno podría preguntarse ipor qué son importantes los procesos pasados e históricos cuando lo que se requiere de las escuelas son estudiantes "alfabetizados cientificamente» que tengan una comprensión de algunos aspectos básicos de ciencias y que les sirva si desean continuar una carrera de ciencias? Esto, en efecto, es un importante objetivo en todo curso de ciencias, pero es importante tener en mente que la mayor parte de los que dejan la escuela no llegarán a científicos. Esta mayoría necesita saber ciencias pero también necesitan apreciar sus limitaciones y su potencial y, para ambos, para los que continuen en ciencias y para los que puede que no, una comprensión de esto y de qué es lo que hacen los científicos, cómo producen conocimientos, y de las presiones intelectuales, económicas, políticas, morales y éticas sobre ellos, sólo puede alcanzarse adecuadamente a través de una perspectiva histórica.

Si vamos a enseñar la ciencia como un proceso y, principalmente, como un proceso de enfrentamiento a problemas/rompecabezas, y conseguir una comprensión de esto a partir de perspectivas tanto actuales como históricas, entonces deben contemplarse cambios grandes y de largo alcance en la enseñanza dé las ciencias en

\section{REFERENCIAS BIBLIOGRÁFICAS}

AYLESWORTH, T.G., 1965, The need for problem-solving. Science Education, Vol. 49, 156.162.

BARNES, D., 1982, Practical Curriculum Study. (Routledge and Kegan Paul: London).

BERNSTEIN, B., 1971, On the classiffication and framing of educational knowledge. En Young, M.F.P. (ed.) Knowledge and Control. (Collier Macmillan: London). la escuela. Como mínimo deberian tenerse en cuenta las áreas fundamentales siguientes:

1. La formación del profesorado: cualquier cambio importante en la dirección de los currículos escolares requiere el perfeccionamiento de los profesores y se necesita un trabajo extensivo con los profesores en activo. Dicho cambio, como se concibe aquí, no obstanie, requeriria también un replanteamiento total de la formación inicial del profesorado.

2. Dirección y organización escolar: se requiriria una flexibilidad en espacio y horario para hacer posible los periodos prolongados de investigación que se lleven a cabo, y que la división tradicional del espacio escolar en celdillas y del tiempo escolar en periodos fijos no permite. Igualmente la integración de las materias que está implícita en mucho de lo que aquí se ha sugerido, requiere una mayor apertura en la arquitectura y en la distribución del tiempo.

3. Exámenes y procedimientos de valoración: también deberian sufrir un considerable replanteamiento y de satrollo si atributos tales como originalidad y creatividad, por ejemplo, tuvieran que reflejarse adecuadamente en cualquier valoración de las características y habilidades de los estudiantes.

4. Sociedad: de la mayor importancia en cualquier cambio curricular es que los padres y la sociedad en general sean conscientes de la necesidad del cambio y lo vean con buenos ojos; y esto requiere desplegar una gran cantidad de buenas relaciones públicas.
BLOUGH, M.G., 1942, Teaching problem-solving to children. Science Teacher, 22-27, (Yearbook of American. Council of Science Teaching, Supplement).

BRANDON, E.P., 1981, Logic in the laboratory. School Science Review, Vol. 62, 762-769.

BROWN, S.A., 1977, A review of the meanings of, and arguments for, integrated science. Studies in Science Edu- 
cation, Vol. 4, 31-62.

BRUNER, J.S., 1962, On Learning. (Harvard University Press: Harvard).

CLOSSET, J.L., FAUCONNET, S., SALTIEL, E. y VIENNOT, L., 1982, Research in science educations at the university VII. European Journal in Science Education. Vol. $4,111-114$.

DEBNEY, B.B., 1971, Creative Problem-solving, Interests in Arts and Science. Unpublished M. Ed. thesis, University of Birmingham.

EGGLESTON, J.F., GALTON, M.J. y JONES, M.E., 1975, Processes and Products of Science Teaching. (The Schools Council: Macmillan, London).

GARRETT, R.M., 1984, Selected Cognitive Styles and Aspects of Their Relationship to Problem-solving: an Empirical Study Using Problems in Physics. Tesis Doctoral no publicada. University of Keele.

GARRETT, R.M., 1987, Issues in science education: problem-solving, creativity and originality. International Journal of Science Education, Vol. 9, 125-137.

GIL PEREZ, D. y MARTÍNEZ TORREGROSA, J., 1983, A Model for problem solving in accordance with scientific methodology European Journal in Science Education, Vol. 5, 447-457.

HOLT, P.N., 1969, A Study of Children's Responses to Open-ended Problems. Tesis Doctoral no publicada. Uni- versity of Southampton.

HUMPHREY, G., 1951, Thinking. (Methuen: London).

KUHN, T.S., 1962, The Structure of Scientific Revolutions. International Encyciopaedia of Unified Science. Foundations of the Unity of Science of Science, Vol. 11, Number 2 .

MEDNICK, S.A., 1962, The associative basis of the creative process. Psychological Review, Vol. 69, 222.232.

PARVES, J.J., BRUNELLE, E.A., 1967, Literature of Creativity Part I. J.C.B., 1.

ROGERS, C.R., 1959, Towards a theory of creativity. In H. Anderson (ed.) Creativity and Cultivation. (Harper \& Row: New York)

RONNING, R.R., MCCURDY, D. y BALLINGER, R., 1984, Individual differences: a third component in problem-solving instruction. Journal of Research in Science Teaching. Vol. 21, 71-82.

STEIN, M.I., 1953, Creativity and cutture. Journal of Psychology, Vol. 36, 311.322.

STOLLBERG, R.J., 1956, Problem-solving, the precious gem in science teaching. Science Teacher, Vol. 23, 225-228.

TURNER, C.G., 1957, Problem-solving a change concept. Science Teacher, Vol. 24, 339-341.

WILSON, J.M., 1977, Practical work in physics in Scottish schools. Schools Science Review, Vol. 58, 783-789 